\title{
Sustained lung inflation in late preterm infants: a randomized controlled trial
}

\author{
D Mercadante ${ }^{1}$, M Colnaghi $^{1}, \mathrm{~V}$ Polimeni ${ }^{1}, \mathrm{E} \mathrm{Ghezzi}^{1}, \mathrm{M}$ Fumagalli ${ }^{1}, \mathrm{D}$ Consonni ${ }^{2}$ and $\mathrm{F} \mathrm{Mosca}^{1}$
}

\begin{abstract}
OBJECTIVE: To assess the need for respiratory support in late preterm infants treated with sustained lung inflation (SLI) at birth. STUDY DESIGN: In this controlled trial, we randomly assigned infants born at $34^{+0}$ to $36^{+6}$ weeks of gestation to receive SLI $\left(25 \mathrm{cmH}_{2} \mathrm{O}\right.$ for $\left.15 \mathrm{~s}\right)$ at birth, followed by continuous positive airway pressure (CPAP) or assistance according to the recommendations of the American Academy of Pediatrics. The primary outcome was the need for any type of respiratory support. The secondary outcomes included neonatal intensive care unit (NICU) admission for respiratory distress and length of stay. The risk ratios (RRs) and 95\% confidence intervals (Cls) of the outcomes were calculated for the SLI group in reference to the control group. RESULTS: A total of 185 infants were enrolled: 93 in the SLI group and 92 in the control group. No difference was found in the need for any type of respiratory support between the infants treated with SLI and the control group (10.6 vs $8.7 \%, \mathrm{RR} 1.24,95 \% \mathrm{Cl} 0.51$ to 2.99). The NICU admission for respiratory distress and the length of stay did not differ between the groups.

CONCLUSION: Providing SLI at birth in late preterm infants does not affect their need for respiratory support.
\end{abstract}

Journal of Perinatology (2016) 36, 443-447; doi:10.1038/jp.2015.222; published online 28 January 2016

\section{INTRODUCTION}

Currently, late preterm births (infants born at 34 to 36 weeks of gestation) account for $\sim 70 \%$ of all preterm births. These infants, which are still physiologically and developmentally immature, have higher risks of morbidity and mortality compared with infants born at term. ${ }^{1,2}$ Because fetal lung maturation occurs late in gestation, respiratory morbidities constitute a major concern for late preterm infants. Anomalies in the process of fetal lung fluid reabsorption and surfactant deficiency are the main factors involved in respiratory disorders of late preterm infants. ${ }^{3-5}$

Some studies have recently investigated the effectiveness of early sustained lung inflation (SLI) in preterm infants to prevent mechanical ventilation. This strategy, consisting of the administration of a positive inflation pressure for a period of time that is significantly more prolonged than the normal inspiratory time (15 to $25 \mathrm{~s}$ ), is considered an alternative to clear fluid-filled lungs and to achieve an early functional residual capacity (FRC). ${ }^{6-12}$

The current evidence is not sufficient to support the efficacy and safety of SLI in preterm infants; furthermore, no reports of its use in late preterm infants are available. The aim of our study was to evaluate the effectiveness of SLI in reducing the need for respiratory support in late preterm infants.

\section{METHODS}

This randomized controlled trial was conducted in a tertiary-level neonatal intensive care unit (NICU) in Milan between September 2013 and June 2014. We enrolled all consecutive inborn infants with a gestational age of 34 to 36 weeks after obtaining parental consent. Infants with major congenital anomalies, or those for whom it was not possible to obtain parental consent, were excluded. The study was approved by the Institutional Review Boards of the Fondazione IRCCS Ca' Granda, Ospedale Maggiore Policlinico, Milan, Italy. Eligible infants were randomly allocated to one of the two following treatment groups using sealed opaque envelopes:

- Group 1 (SLI group) - Infants assigned to this group were placed at birth under a radiant heat source and, after oropharyngeal and nasal suctioning, a prophylactic pressure-controlled $\left(25 \mathrm{cmH}_{2} \mathrm{O}\right)$ inflation was sustained for $15 \mathrm{~s}$ using a neonatal mask and a T-piece ventilator, followed by the delivery of $5 \mathrm{cmH}_{2} \mathrm{O}$ CPAP. In the following 6 to $10 \mathrm{~s}$, CPAP was discontinued in the absence of signs of inadequate respiratory effort (that is, apnea or gasping) or heart rate $>100$ beats per min (b.p.m.). In the presence of signs of inadequate respiratory effort and/or whenever the heart rate was between 60 and 100 b.p.m. despite CPAP, a SLI maneuver with the same parameters was repeated. If the heart rate was $<100$ b.p.m. after the second SLI maneuver, the infant was resuscitated according to the recommendations of the American Academy of Pediatrics (AAP).

- Group 2 (Control group) - Infants assigned to this group were assisted according to the recommendations of the AAP, providing the initial steps in stabilization (warming and maintaining normal temperature, positioning, clearing secretions only if copious and/or obstructing the airway, drying, and stimulating). We progressed beyond the initial steps in the presence of inadequate respiratory effort (defined as above) and/ or a $\mathrm{HR}<100$ b.p.m. according to the AAP recommendations.

In both groups, respiratory support in the delivery room was given using a T-piece ventilator (Neopuff Infant T-Piece Resuscitator, Fisher \& Paykel, Auckland, New Zealand). To avoid pressure leakage, we used a properly sized neonatal mask that adequately covered both the mouth and nostrils of the infants (size 0 or 1 face mask, Ambu UltraSeal, Ballerup, Denmark). The flow rate was set at 8 to $10 \mathrm{Imin}^{-1}$. Infants in both groups who developed respiratory distress were admitted to the NICU; during an observation period of $30 \mathrm{~min}$, the respiratory rate, pulse oximeter oxygen saturation $\left(\mathrm{SpO}_{2}\right)$, and the severity of respiratory distress measured using the Silverman score were monitored. The need for oxygen supplementation was assessed to maintain an $\mathrm{SpO}_{2}$ between 88 and $95 \%$. At the end of

\footnotetext{
${ }^{1} \mathrm{NICU}$, Department of Clinical Sciences and Community Health, Fondazione IRCCS Cà Granda Ospedale Maggiore Policlinico, Università degli Studi di Milano, Milan, Italy and ${ }^{2}$ Epidemiology Unit, Fondazione IRCCS Ca' Granda Ospedale Maggiore Policlinico Milano, Università degli Studi di Milano, Milan, Italy. Correspondence: Dr D Mercadante, NICU,

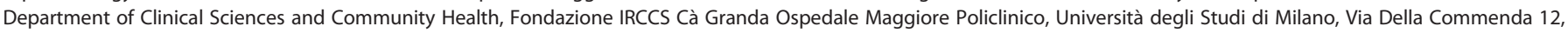
Milan, MI 20122, Italy.
}

E-mail: domenica.mrc@hotmail.it

Received 29 July 2015; revised 19 November 2015; accepted 17 December 2015; published online 28 January 2016 
the observation period, if the infant needed a fraction of inspired oxygen $\left(\mathrm{FiO}_{2}\right) \geqslant 0.30$ to maintain an $\mathrm{SpO}_{2}$ between 88 and $95 \%$ and/or the Silverman score was $\geqslant 5$, a non-invasive respiratory support (nasal CPAP of 5 to $6 \mathrm{cmH}_{2} \mathrm{O}$, Infant Flow SiPAP System - Viasys Healthcare, Yorba Linda, California, USA; High Flow Therapy, flow 4 to 6 LPM, Vapotherm, Stevensville, Maryland, USA) was initiated. Infants requiring $\mathrm{FiO}_{2}>0.40$ to maintain an $\mathrm{SpO}_{2}$ of 88 to $93 \%$ and/or with a Silverman score $>6$ were treated with surfactant $(200 \mathrm{mg} / \mathrm{kg}$; Curosurf, Chiesi Farmaceutici SpA, Parma, Italy) with the INtubation-SURfactant administration-EXtubation procedure. Infants were intubated when the $\mathrm{pH}$ was $<7.20$ with partial pressure of carbon dioxide $\left(\mathrm{PaCO}_{2}\right)>70 \mathrm{mmHg}$ or if $\mathrm{FiO}_{2}>0.40$ after surfactant treatment was needed to maintain an $\mathrm{SpO}_{2}$ of 88 to $93 \%$, or if infants had frequent episodes of apnea ( $>4$ episodes in $1 \mathrm{~h}$ or $>2$ episodes requiring bag-and-mask ventilation) despite adequate noninvasive support. Infants were extubated when they met all of the following criteria: $\mathrm{FiO}_{2}<0.40$, mean airway pressure $<6 \mathrm{cmH}_{2} \mathrm{O}$, $\mathrm{PaCO}_{2}<65 \mathrm{mmHg}$ with a $\mathrm{pH}>7.20$, and $\mathrm{PaO}_{2}>50 \mathrm{mmHg}$.

The primary outcome of the study was to evaluate the effectiveness of SLI in reducing the need for respiratory support in late preterm infants compared with control infants. Secondary outcomes were NICU admission, NICU admission for respiratory disease, length of stay, and exclusive breastfeeding at discharge.

Perinatal and neonatal data were collected for each infant as follows: gestational age, birth weight, sex, mode of delivery (vaginal or cesarean section), Apgar at 1 st and 5th minute, antenatal steroid treatment, main diseases of pregnancy, NICU admission, Silverman score, diagnosis of respiratory disease (respiratory distress syndrome, transient tachypnea of the newborn, pneumonia, and persistent pulmonary hypertension), type of respiratory support, total length of respiratory support, surfactant administration, air leak syndromes, exclusive breastfeeding at discharge, length of stay, and mortality. The diagnosis of respiratory distress syndrome was based on the occurrence of typical signs (need for oxygen supplementation, tachypnea, intercostal retractions, and grunting), and findings on chest radiographs (decreased lung expansion, reticulogranular pattern of the lung, and air bronchograms), and the exclusion of other causes of respiratory failure. The diagnosis of transient tachypnea of the newborn was based on chest radiographs indicative of retained fluid (for example, streakiness, fluid in fissure), an oxygen requirement $>40 \%$, and spontaneous improvement over two to five days. Pneumonia was defined by an abnormal chest radiograph in the presence of two of the following: extra-pulmonary clinical signs of sepsis, abnormal non-specific (screening) laboratory tests, positive blood culture, positive tracheal culture, or perinatal history indicating exposure (prolonged rupture, maternal fever, so on). Diagnosis of persistent pulmonary hypertension was based on an echocardiogram.

The study was not blinded. The decision to start respiratory support was made by clinicians other than the investigators involved in the study according to specific guidelines, and researchers assessing the study end points were blinded to the nature of the study treatments. The staff involved in the study is a skilled team of specialists in neonatal resuscitation and the SLI maneuver is a well-established procedure at our institution and has been used in previous clinical trials. Comprehensive education and training were undertaken to ensure technical proficiency and protocol compliance through audits with clinical personnel.

\section{Statistical analysis}

From 2009 to 2012, 15\% of 2503 late preterm infants born at our center needed respiratory assistance (invasive or non-invasive ventilation). In calculating the sample size, we followed the confidence interval approach. ${ }^{13-14}$ We calculated a sample size of $92 \times 2$ newborns assuming a reduction in the need for respiratory assistance in this population from 15 to $5 \%$, that is, a risk ratio of 0.33 with a $95 \%$ confidence interval from 0.13 to 0.88 . We compared the characteristics between the SLI and the control group using Student's $t$-test or the Mann-Whitney test for quantitative variables and the $x^{2}$-test for categorical variables. We calculated the risk ratios and $95 \%$ confidence intervals of primary and secondary outcomes for the SLI group in reference to the control group. All analyses were performed with version 9.2 SAS software (SAS Institute, Cary, NC, USA, 1999).

\section{RESULTS}

In the period between September 2013 and June 2014, we enrolled a total of 185 late preterm infants. The number of infants deemed eligible for the study and the number of whom were randomly assigned to receive the SLI procedure or standard assistance in the delivery room are shown in Figure 1. The baseline characteristics of infants at birth were equally distributed between the two groups (Table 1). More than $90 \%$ of infants were born by cesarean section in both groups; an emergent cesarean section, in the absence of labor, was performed in $21 \%$ of cases. Twins accounted for over $50 \%$ of the study population.

Table 2 shows the primary and secondary outcomes of the study. There was no difference in the need for any type of respiratory support between the infants treated with SLI at birth and the control group ( 10.6 vs $8.7 \%, P=0.64)$. Six $(60 \%)$ out of the 10 infants in the SLI group who required respiratory support had a diagnosis of respiratory distress syndrome and four (40\%) had a diagnosis of transient tachypnea of the newborn. Eight infants in the control group required respiratory support, two (25\%) with a diagnosis of respiratory distress syndrome, and six (75\%) with a

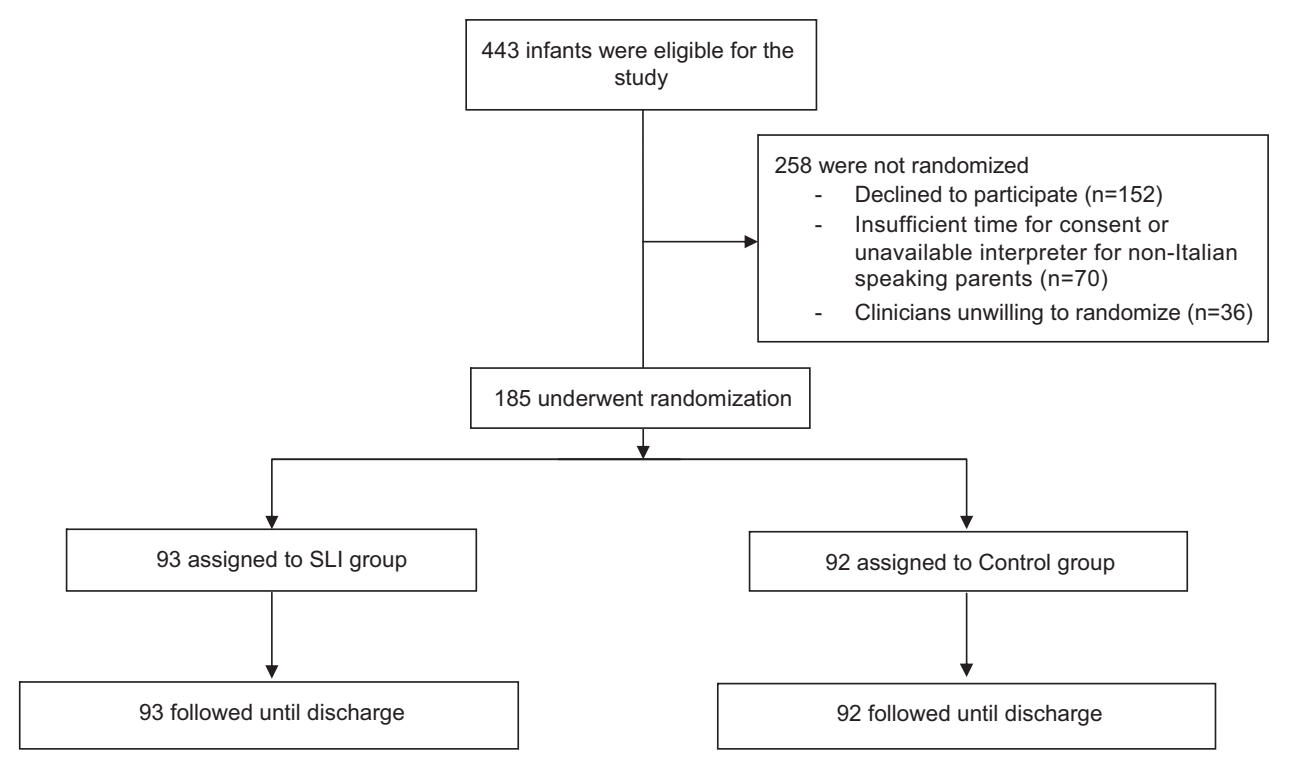

Figure 1. Diagram showing the flow of participants through the trial. 
Table 1. Characteristics of infants in SLI and Control group

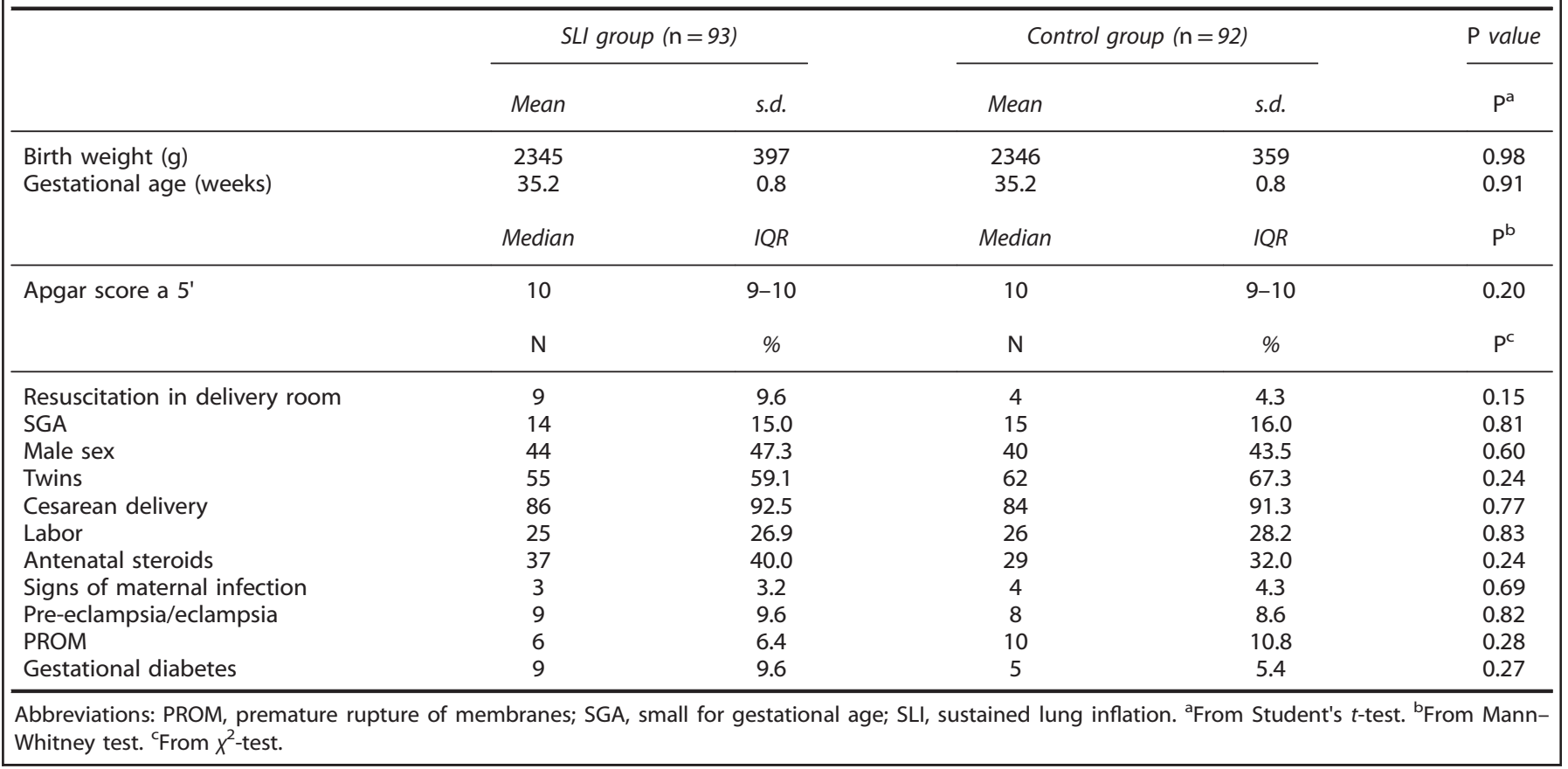

Table 2. Primary and secondary outcomes

\begin{tabular}{|c|c|c|c|c|c|c|c|}
\hline & $\mathrm{N}$ & $\%$ & $\mathrm{~N}$ & $\%$ & $R R$ & $95 \% \mathrm{Cl}$ & $\mathrm{P}$ value \\
\hline \multicolumn{8}{|l|}{ Primary outcome } \\
\hline Need for any type of respiratory support & 10 & 10.7 & 8 & 8.7 & 1.24 & $0.51-2.99$ & 0.64 \\
\hline NICU admission & 37 & 39.8 & 27 & 29.4 & 1.35 & $0.90-2.03$ & 0.14 \\
\hline NICU admission for respiratory disease & 17 & 18.3 & 10 & 10.9 & 1.68 & $0.81-3.47$ & 0.15 \\
\hline Length of stay ( $>7$ days) & 42 & 45.1 & 38 & 41.3 & 1.09 & $0.78-1.52$ & 0.59 \\
\hline Exclusive breastfeeding at discharge & 25 & 26.9 & 17 & 18.5 & 1.45 & $0.84-2.50$ & 0.17 \\
\hline
\end{tabular}

diagnosis of transient tachypnea of the newborn. Similarly, NICU admission, NICU admission for respiratory distress, length of stay, and exclusive breastfeeding at discharge did not differ between the two groups. All of the 185 infants were followed until discharge, and none died during the in-hospital stay.

Three infants (3.2\%) developed a pneumothorax in the SLI group compared with no infants in the control group $(P=0.08)$. The clinical characteristics of infants who developed pneumothorax are detailed in Table 3. None of these infants required vigorous resuscitation at any time. In two cases, a chest tube was placed for tension pneumothorax.

\section{DISCUSSION}

This study evaluated the effectiveness of SLI at birth in reducing the need for respiratory support in late preterm infants compared with controls. Our results demonstrated that a prophylactic SLI at birth does not affect the need for respiratory support in late preterm infants.
Respiratory assistance in the delivery room is an aspect of respiratory care in preterm infants that has been thoroughly investigated in recent years. The critical event is the aeration of the fluid-filled lung; at birth, a pressure gradient is required to overcome airflow resistance and move lung fluid distally through the airway to the terminal airspaces, where the opening pressure is necessary to overcome the surface tension and open the alveoli. While term infants begin to establish the FRC with their first breath after birth, preterm infants are hampered because of poor respiratory drive, limited muscle strength, high chest wall compliance, and surfactant system immaturity. ${ }^{15}$ The SLI has been suggested as an additional approach to promote lung fluid clearance and aeration, as it mimics the first breaths of life in healthy term infants that are characterized by a prolonged inspiratory time due to a long time constant of the fluid-filled lung, a prolonged expiratory phase and high inflation pressure. The effect of SLI as an initial 'prolonged' inflation is to move the air/liquid interface distally, with direct lung aeration and clearance of lung fluid from the major airways immediately after birth. This 
Table 3. Clinical characteristics of infants who developed pneumothorax

\begin{tabular}{lccccccccc}
\hline Treatment arm GA (wk) & BW $(g)$ & $\begin{array}{c}\text { Antenatal } \\
\text { steroids }\end{array}$ & Labor & $\begin{array}{c}\text { Mode of } \\
\text { delivery }\end{array}$ & $\begin{array}{c}\text { Age at PNX } \\
(h)\end{array}$ & $\begin{array}{c}\text { Chest tube } \\
\text { placement }\end{array}$ & $\begin{array}{c}\text { Respiratory support } \\
\text { at PNX }\end{array}$ & $\begin{array}{c}\text { Status at } \\
\text { discharge }\end{array}$ \\
\hline SLI & 35 & 2370 & No & No & CS & 36 & Yes & nCPAP bilevel \\
SLI & 36 & 2410 & No & No & CS & 13 & Yes & nCPAP bilevel & Alive \\
SLI & 36 & 2845 & No & No & CS & 48 & No & nCPAP & Alive \\
\hline
\end{tabular}

Abbreviations: BW, birth weight; CS, cesarean section; GA, gestational age; nCPAP, nasal continuous positive airway pressure; PNX, pneumothorax; SLI, sustained lung inflation.

process is completed by interstitial lymphatic and venous reabsorption in the following hours. ${ }^{16-17}$

The effect of SLI to facilitate lung aeration and improve postnatal ventilation has been investigated in animal and human studies. $^{6-12,18-22}$ In preterm infants, the evidence to support its efficacy and safety remains limited, and no reports of its use in late preterms are available. We chose to study this population of preterm infants as they account for an increasing proportion of respiratory-associated short-term morbidities with a need for specialized care and prolonged hospital stays. ${ }^{5,23,24}$

Colin et al. $^{25}$ suggested that the functional consequences of late preterm birth include difficulty in protecting FRC, vulnerability to airway collapse, and increased airway resistance. Moreover, rapid clearance of the fetal lung fluid plays a key role in the transition to air breathing; after late preterm birth, this process can be delayed because this is dependent upon developmentally regulated epithelial sodium channels, especially in the absence of spontaneous labor and related hormonal changes, as frequently occurs in elective cesarean sections. ${ }^{26}$ Because the delayed absorption of lung fluid is an important cause of respiratory failure in late preterms, we speculated that SLI could reduce the need for respiratory care in these infants, although no previous experimental or human studies on the effectiveness of SLI in this population are available.

We estimated a sample size by considering a reduction in the need for respiratory support from 15 to $5 \%$ and a number needed to treat of 10 , which is clinically relevant. We think that a less significant reduction would not justify a prophylactic SLI whose risks and benefits are not yet completely known.

We applied the sustained inflations using a face mask. As recently demonstrated by van Vonderen et al., ${ }^{27}$ the effect of SLI on FRC gain may be impaired by substantial leakiness and a limited volume may consequently be delivered. In studies that reported the efficacy of SLI in creating inspiratory volume and FRC, the sustained inflation was applied via an endotracheal tube, bypassing the glottis and minimizing leaks.,22 However, van Vonderen et al. ${ }^{27}$ observed that, even in infants in whom no leak was present, the delivered volumes achieved by the sustained inflation were very low. It is possible that closure of the vocal cords in both breathing (that is, between breaths) and apneic infants could cause obstruction; furthermore, obstruction occurring at the pharyngeal level by a relatively large tongue or obstruction of the nose and mouth could occur with improper mask technique. In our study, lung volumes were not recorded, but it is reasonable to believe that the problems described by van Vonderen et al. ${ }^{27}$ may have influenced our results. We believe that it would be helpful in further studies to evaluate lung mechanics during the SLI and to assess the optimal parameters of the maneuver (time and pressure), actual lung recruitment and its variations over time.

There are some limitations to our study. We performed SLI in all late preterm infants; therefore, we also performed SLI in infants with appropriate lung fluid reabsorption and adequate FRC. Although this approach was used in the largest multicenter randomized controlled trial to date, ${ }^{21}$ in late preterm infants with adequate respiratory effort and partially aerated lungs, SLI at birth could make them more susceptible to over-distention and air leaks. In our study, pneumothorax occurred in $3.2 \%(n=3)$ of infants in the SLI group compared with no infants in the control group $(P=0.08)$; all pneumothorax events occurred beyond the first $12 \mathrm{~h}$ of life. This result should be taken into consideration, although the difference was not statistically significant and the number of cases is limited. In the recent study by Lista et al., ${ }^{21}$ most of the pneumothoraces occurred beyond the first $48 \mathrm{~h}$ of life, rather than soon after the SLI maneuver. A possible reason for this finding as suggested by Keszler ${ }^{28}$ could be that the late pneumothoraces could be related to the reluctance to intubate and provide surfactant in the intervention group of this unblinded study in which the need for mechanical ventilation was the primary outcome, rather than the intervention itself. No other studies have shown an increase in air leaks in the intervention group treated with $\mathrm{SLI}^{29}$

Another limitation of the study is that it is a non-blinded study and a single site trial. We tried to minimize this bias through adequate training of care providers, by using clinicians who had the decision to start respiratory support other than the investigators involved in the study, and by blinding the researchers that assessed the study end points to the nature of the study treatments.

\section{CONCLUSION}

Delivery room sustained inflation is an attractive concept in the respiratory assistance of preterm infants at birth. To the best of our knowledge, no reports of its use in late preterm infants are available. Our results demonstrated that prophylactic SLI at birth does not reduce the need for respiratory support in these preterm infants. Possible future trials should exclude infants with adequate respiratory effort and focus on late preterm infants who require resuscitation intervention at birth due to inadequate respiratory effort and thus likely to benefit from recruitment maneuver. Moreover, trials should include close monitoring of adverse events, especially with regard to the risk of overdistension and air leak syndromes. Associated measures of respiratory mechanics could be useful to evaluate the efficacy and individualize the SLI maneuver at birth.

\section{CONFLICT OF INTEREST}

The authors declare no conflict of interest.

\section{ACKNOWLEDGEMENTS}

The authors have no financial relationships relevant to this article to disclose. No honorarium, grant or other form of payment was given to any of the authors to produce the manuscript.

\section{REFERENCES}

1 Shapiro-Mendoza CK, Lackritz EM. Epidemiology of late and moderate preterm birth. Semin Fetal Neonatal Med 2012; 17(3): 120-125.

2 Kugelman A, Colin AA. Late preterm infants: near term but still in a critical developmental time period. Pediatrics 2013; 132(4): 741-751.

3 Gouyon JB, lacobelli S, Ferdynus C, Bonsante F. Neonatal problems of late and moderate preterm infants. Semin Fetal Neonatal Med 2012; 17(3): 146-152. 
4 De Luca R, Boulvain M, Irion O, Berner M, Pfister RE. Incidence of early neonatal mortality and morbidity after late-preterm and term cesarean delivery. Pediatrics 2009; 123(6): e1064-e1071.

5 Consortium on safe labour, Hibbard JU, Wilkins I, Sun L, Gregory K, Haberman S et al. Respiratory morbidity in late preterm births. JAMA 2010; 304(4): 419-425.

6 Lindner W, Vossbeck S, Hummler H, Pohlandt F. Delivery room management of extremely low birth weight infants: spontaneous breathing or intubation? Pediatrics 1999; 103 (5 Pt 1): 961-967.

7 te Pas AB, Siew M, Wallace MJ, Kitchen MJ, Fouras A, Lewis RA et al. Establishing functional residual capacity at birth: the effect of sustained inflation and positive end-expiratory pressure in a preterm rabbit model. Pediatr Res 2009; 65(5): 537-541.

8 te Pas AB, Siew M, Wallace MJ, Kitchen MJ, Fouras A, Lewis RA et al. Effect of sustained inflation length on establishing functional residual capacity at birth in ventilated premature rabbits. Pediatr Res 2009; 66(3): 295-300.

9 Polglase GR, Tingay DG, Bhatia R, Berry CA, Kopotic RJ, Kopotic CP et al. Pressureversus volume-limited sustained inflations at resuscitation of premature newborn lambs. BMC Pediatr 2014; 14: 43.

10 te $\mathrm{Pas} A B$, Walther FJ. A randomized, controlled trial of delivery room respiratory management in very preterm infants. Pediatrics 2007; 120(2): 322-329.

11 Lindner W, Hoegel J, Pohlandt F. Sustained pressure-controlled inflation or intermittent mandatory ventilation in preterm infants in the delivery room? A randomized, controlled trial on initial respiratory support via nasopharyngeal tube. Acta Paediatr 2005; 94(3): 303-309.

12 Lista G, Fontana P, Castoldi F, Cavigioli F, Dani C. Does sustained lung inflation at birth improve outcome of preterm infants at risk for respiratory distress syndrome? Neonatology 2011; 99(1): 45-50.

13 Bland M. The tyranny of power: is there a better way to calculate sample size? BMJ 2009; 339: b3985.

14 Goodman SN, Berlin JA. The use of predicted confidence intervals when planning experiments and the misuse of power when interpreting results. Ann Intern Med 1994; 121: 200-206.

15 Te Pas AB, Davis PG, Hooper SB et al. From liquid to air: breathing after birth. J Pediatr 2008; 152: 607-611.

16 Vento M, Lista G. Managing preterm infants in the first minutes of life. Paediatr Respir Rev 2015; 16(3): 151-156.
17 Hooper SB, Siew ML, Kitchen MJ, te Pas AB. Establishing functional residual capacity in the non-breathing infant. Semin Fetal Neonatal Med 2013; 18(6): 336-343.

18 Hillman NH, Kemp MW, Noble PB, Kallapur SG, Jobe AH. Sustained inflation at birth did not protect preterm fetal sheep from lung injury. Am J Physiol Lung Cell Mol Physiol 2013; 305(6): L446-L453.

19 Klingenberg C, Sobotka KS, Ong T, Allison BJ, Schmolzer GM, Moss TJ et al. Effect of sustained inflation duration; resuscitation of near-term asphyxiated lambs. Arch Dis Child Fetal Neonatal Ed 2013; 98: F222-F227.

20 Sobotka KS, Hooper SB, Allison BJ, Te Pas AB, Davis PG, Morley CJ et al. An initial sustained inflation improves the respiratory and cardiovascular transition at birth in preterm lambs. Pediatr Res 2011; 70(1): 56-60.

21 Lista G, Boni L, Scopesi F, Mosca F, Trevisanuto D, Messner H et al. Sustained lung inflation at birth for preterm infants: a randomized clinical trial. Pediatrics 2015; 135(2): e457-e464.

22 Vyas H, Milner A, Hopkin I, Boon A. Physiologic responses to prolonged and slowrise inflation in the resuscitation of the asphyxiated newborn infant. J Pediatr 1981; 99(4): 635-639.

23 Teune MJ, Bakhuizen S, Gyamfi Bannerman C, Opmeer BC, van Kaam $\mathrm{AH}$ van Wassenaer AG et al. A systematic review of severe morbidity in infants born late preterm. Am J Obstet Gynecol 2011; 205(4): 374.e1-9.

24 Pike KC, Lucas JS. Respiratory consequences of late preterm birth. Paediatr Respir Rev 2014; 16 (3): 182-188.

25 Colin AA, McEvoy C, Castile RG. Respiratory morbidity and lung function in preterm infants of 32 to 36 weeks' gestational age. Pediatrics 2010; 126(1): $115-128$.

26 Jain L, Eaton DC. Physiology of fetal lung fluid clearance and the effect of labor. Semin Perinatol 2006; 30(1): 34-43.

27 van Vonderen JJ, Hooper SB, Hummler HD, Lopriore E, te Pas AB. Effects of a sustained inflation in preterm infants at birth. J Pediatr 2014; 165(5): 903-8.e1.

28 Keszler M. Sustained inflation during neonatal resuscitation. Curr Opin Pediatr 2015; 27(2): 145-151.

29 Schmölzer GM, Kumar M, Aziz K, Pichler G, O'Reilly M, Lista G et al. Sustained inflation versus positive pressure ventilation at birth: a systematic review and meta-analysis. Arch Dis Child Fetal Neonatal Ed 2015; 100 (4): F361-F368. 